\title{
Vacuum Phenomenon of the Sacroiliac Joint: Correlation with Sacropelvic Morphology
}

\author{
Yoichiro Takata, Kosaku Higashino, Masatoshi Morimoto, Toshinori Sakai, \\ Kazuta Yamashita, Mitusnobu Abe, Akihiro Nagamachi, Koichi Sairyo \\ Department of Orthopedics, Tokushima University, Tokushima, Japan
}

\begin{abstract}
Study Design: A radiologic study of sacropelvic morphology and vacuum phenomenon of sacroiliac joint in subjects unrelated to low back pain.

Purpose: The aim of this study is to describe the relationship between sacropelvic morphology and vacuum phenomenon of the sacroiliac joint.

Overview of Literature: Lumbopelvic alignment and sacropelvic morphology are associated with the pathomechanisms of various spinal disorders. The vacuum phenomena of the sacroiliac joint (SJVP) are often observed in clinical practice, but the relationships between these phenomena and sacropelvic morphology have not been investigated. This study examined the prevalence of SJVP in computed tomography (CT) images and the relationship between sacropelvic morphology and SJVP.

Methods: We analyzed multiplanar CT images of 93 subjects (59 men, 34 women). Pelvic incidence (PI), pelvic tilt (PT), sacral slope (SS), and lumbar lordosis (LL) were measured using the three-dimensional reconstruction method. The prevalence of SJVP in multiplanar CT images were reviewed. Roland-Morris Disability Questionnaire (RDO) scores and the modified Japanese Orthopedic Association (JOA) score, which focuses on subjective symptoms and restriction of activities of daily living, were also obtained from all the subjects.

Results: Thirty-six of the 93 subjects had SJVP (39\%), with marked female predominance (91\% women, 8.5\% men). Men with SJVP had significantly lower PI than men without SJVP $\left(35.1^{\circ}\right.$ vs. $\left.46.3^{\circ}, p<0.05\right)$. There was no correlation between SJVP and the modified JOA or RDQ scores.

Conclusions: These data suggest that differences in sacropelvic morphology can influence the biomechanical environment and contribute to SJVP in men. Presence of SJVP did not affect JOA or RDQ scores.
\end{abstract}

Keywords: Sacroiliac joint; Lumbosacral region; Vacuum; Pelvis

\section{Introduction}

Recent studies of patients with spinal deformities have demonstrated that global spinal misalignment in the sagittal plane is a strong predictor of disability [1-4]. Global spinal alignment is described by lumbopelvic parameters, characterized by both spinal thoracic kyphosis (TK) and lumbar lordosis (LL), and sacropelvic parameters of pelvic incidence (PI), pelvic tilt (PT), and sacral slope (SS). PI is a key pelvic parameter as it regulates the sagittal curves and determines the degree of lumbar lordosis [5]. It is an anatomic parameter that is thought to remain unchanged

Received Nov 3, 2015; Revised Nov 27, 2015; Accepted Nov 27, 2015

Corresponding author: Yoichiro Takata

Department of Orthopedics, Tokushima University, 3-18-15 Kuramoto-cho, Tokushima 770-8503, Japan

Tel: +81-88-633-7240, Fax: +81-88-0-633-0178, E-mail: yoichiro76@gmail.com 
during the patient's lifetime, while PT and SS are positional parameters that are influenced by position of the patient.

The vacuum phenomenon (VP) refers to collection of gas within the joint space [6]. Intervertebral VP is usually associated with degenerative disc disease, which can be associated with low back pain. Vacuum phenomenon of the sacroiliac joint (SJVP) is often observed in clinical practice. Its occurrence suggests that the joint is mobile but the actual mobility of the sacroiliac joint (SIJ) is quite small [7]. The significance of SJVP in patients with low back pain has been debated, and there have only been few studies on the SJVP and its clinical relevance $[8,9]$.

Here we investigated the prevalence of SJVP on abdominopelvic computed tomography (CT) images and analyzed the relationships between this phenomenon and sacropelvic morphology or clinical symptoms.

\section{Materials and Methods}

Approval from the Institutional Review Boards of the participating institutions was obtained prior to the study. A total of 100 subjects (65 men, 35 women) who received abdominal and pelvic CT for reasons unrelated to low back pain between 2006 and 2011 were enrolled. Seven subjects with transitional vertebrae or metastatic pelvic tumor were excluded. The modified Japanese Orthopedic Association (JOA) score (mJOA) that focuses on subjective symptoms and restriction of activities of daily living was assessed, and the Roland-Morris Disability Questionnaire (RDQ) was administered to the participants.

\section{Identification of SJVP}

Abdominopelvic CT images were obtained in $1.0 \mathrm{~mm}$ thick axial slices. Images were reviewed at bone (window, 2200; level, 200) window setting. The presence of gas within the SIJ on axial slices parallel to the sacral endplate was considered to be a case of VP. Subjects were divided into groups according to the presence of SJVP (VP+ and VP-groups).

\section{Radiographic measurement of lumbopelvic parameters}

CT scan data were imported into Acquarius NET ver. 1.6 software (TeraRecon, San Mateo, CA, USA) for analysis. PI, PT, SS, and LL were measured from multiplanar CT images (Fig. 1).

\section{Statistical analysis}

For each parameter, differences between the groups were evaluated by the unpaired $t$ test, Mann-Whitney test, or chi-square test as appropriate. A $p<0.05$ was considered statistically significant.

\section{Results}

The characteristics of the subjects are shown in Table 1. There was no significant differences in mean age, mJOA score, or RDQ score between male and female subjects.

\section{Vacuum phenomenon of the SIJ}

There were 36 subjects with SJVP (VP+group) and 57

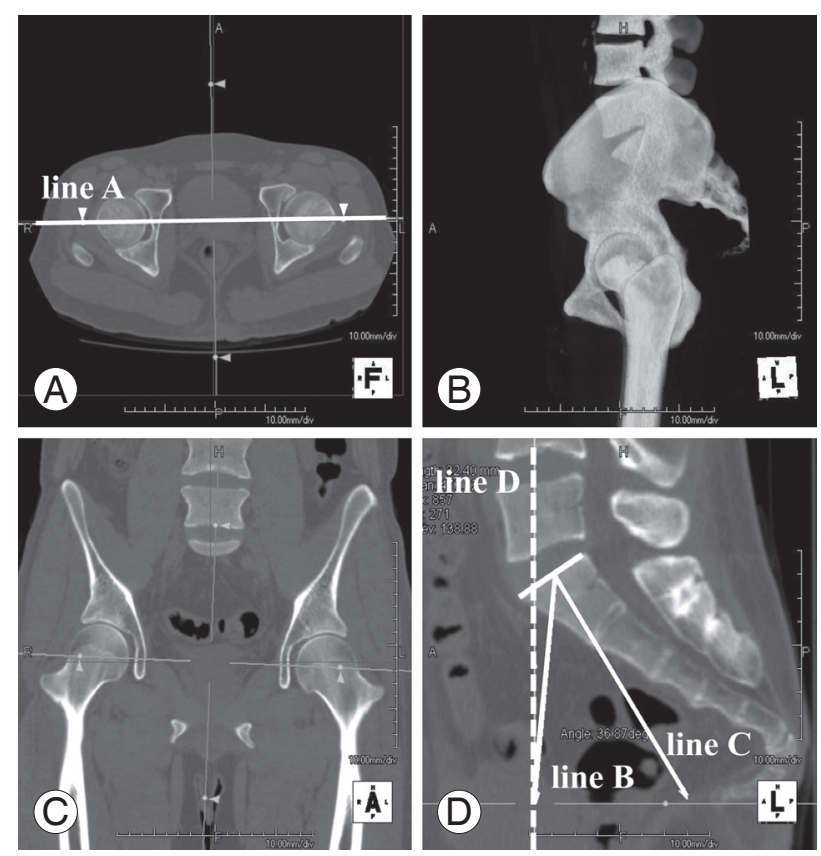

Fig. 1. Radiographic measurement of lumbopelvic parameters. In the axial and coronal view (A, C, respectively), the line connecting the two centers of bilateral femoral heads was considered the "true" bicoxofemoral axis (line A). (B) showing three dimensional model of lumbopelvic region. In the sagittal view (D), the angle between the line from the "true" bicoxofemoral axis to the center of the sacral endplate (line B) and the orthogonal line to the sacral endplate (line C) was measured as the "true" PI. PT was measured as the angle between the "provisional" vertical line (dashed line D) and line B. Pl is equal to the sum of SS and PT. LL was measured between the cranial endplates of L1 and S1. PI, pelvic incidence; PT, pelvic tilt; SS, sacral slope; LL, lumbar lordosis. 
Table 1. Subjects' demographic characteristics

\begin{tabular}{|c|c|c|c|c|}
\hline Characteristic & Overall & Male & Female & $p$-value \\
\hline No. of subjects & 93 & 59 & 34 & - \\
\hline Age (average $\pm S D$ ) & $63.5 \pm 9.7$ & $63.9 \pm 9.7$ & $62.7 \pm 9.6$ & $N S^{a)}$ \\
\hline Modified JOA score & $19.8 \pm 3.2$ & $19.9 \pm 3.1$ & $19.6 \pm 3.5$ & $N S^{a)}$ \\
\hline RD0 & $1.29 \pm 3.2$ & $0.89 \pm 2.2$ & $1.97 \pm 4.4$ & $N S^{a)}$ \\
\hline
\end{tabular}

SD, standard deviation; NS, not significant; JOA, Japanese Orthopedic Association; RDQ, Roland-Morris Questionnaire.

${ }^{\text {a) }}$ Two-tailed $t$-test.

Table 2. Prevalence of SJVP and relationship of SJVP with radiographic parameters and results of questionnaires in overall subjects

\begin{tabular}{|c|c|c|c|}
\hline Overall & SJVP+ $(n=36)$ & SJVP- $(n=57)$ & $p$-value \\
\hline Male & 5 & 54 & $<0.01^{\text {a) }}$ \\
\hline Female & 31 & 3 & \\
\hline Age (average $\pm S D$ ) & $64.4 \pm 9.6$ & $62.9 \pm 9.8$ & $N S^{b)}$ \\
\hline PI (average $\pm S D$ ) & $46.6 \pm 10.9$ & $46.3 \pm 7.0$ & $N S^{b)}$ \\
\hline PT (average $\pm S D$ ) & $10.9 \pm 7.2$ & $9.5 \pm 4.4$ & $N S^{b)}$ \\
\hline SS (average $\pm S D$ ) & $35.7 \pm 8.9$ & $36.9 \pm 5.9$ & $N S^{b)}$ \\
\hline LL (average $\pm S D$ ) & $40.1 \pm 10.7$ & $39.1 \pm 7.9$ & $N S^{b)}$ \\
\hline Modified JOA score & $19.2 \pm 4.0$ & $19.8 \pm 3.2$ & $N S^{b)}$ \\
\hline $\mathrm{RDO}$ & $2.4 \pm 4.7$ & $1.3 \pm 3.2$ & $N S^{b)}$ \\
\hline
\end{tabular}

SJVP, vacuum phenomenon of the sacroiliac joint; SD, standard deviation; NS, not significant; PI, pelvic incidence; PT, pelvic tilt; SS, sacral slope; LL, lumbar lordosis; JOA, Japanese Orthopedic Association; RDQ, Roland-Morris Questionnaire.

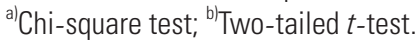

Table 3. Comparison of radiographic parameters between SJVP+ group and SJVP-group in male subjects

\begin{tabular}{|c|c|c|c|}
\hline Male & SJVP+ $(n=5)$ & SJVP- $(n=54)$ & $p$-value \\
\hline PI (average $\pm S D$ ) & $35.1 \pm 4.5$ & $46.3 \pm 7.9$ & $<0.01^{\text {a) }}$ \\
\hline PT (average $\pm S D$ ) & $5.7 \pm 4.5$ & $9.3 \pm 4.3$ & $N S^{a)}$ \\
\hline SS (average $\pm S D$ ) & $29.4 \pm 3.0$ & $36.9 \pm 6.0$ & $<0.01^{\text {a) }}$ \\
\hline $\mathrm{LL}$ (average $\pm S D$ ) & $33.1 \pm 6.3$ & $38 . \pm 7.9$ & $N S^{a)}$ \\
\hline
\end{tabular}

SJVP, vacuum phenomenon of the sacroiliac joint; SD, standard deviation; PI, pelvic incidence; NS, not significant; PT, pelvic tilt; SS, sacral slope; LL; lumbar lordosis.

a) Mann-Whitney U test.

without SJVP (VP-group) (Table 2). There was no correlation between the presence of SJVP and either mJOA score or RDQ. The prevalence of SJVP differed significantly between men and women $(8.5 \%$ vs. $91 \%, p<0.01)$ with female predominance. The lumbopelvic parameters did not differ significantly between VP+ and VP-groups. However, in male subjects, PI and SS were significantly lower in VP+ subjects than in VP-subjects $(p<0.01)$ (Table 3). On the other hand, the presence of SJVP did not affect lumbopelvic parameters in female subjects (Table 4).

\section{Discussion}

The SIJ transmits axial forces generated through the spinal column caudally to the lower limbs [10]. It is a true diarthrodial joint, consisting of a joint cavity, two articular surfaces covered by articular cartilage, and a joint capsule. The long axis of the SIJ is tilted forward at a $50^{\circ}$ angle, and outward at a $20^{\circ}$ angle from the vertical line, opening outward at a $16^{\circ}$ angle on the horizontal plane. The SIJ surface is flat and oriented nearly parallel to the plane 
Table 4. Comparison of radiographic parameters between SJVP+ group and SJVP- group in female subjects

\begin{tabular}{|c|c|c|c|}
\hline Female & SJVP+ $(n=31)$ & SJVP- $(n=3)$ & $p$-value \\
\hline PI (average \pm SD) & $48.5 \pm 10.5$ & $47.6 \pm 9.7$ & $N S^{a)}$ \\
\hline PT (average $\pm S D$ ) & $11.7 \pm 7.2$ & $12.2 \pm 5.6$ & $N S^{a)}$ \\
\hline SS (average $\pm S D$ ) & $36.7 \pm 9.1$ & $35.4 \pm 4.1$ & $N S^{a)}$ \\
\hline LL (average $\pm S D$ ) & $41.2 \pm 10.9$ & $42.1 \pm 7.2$ & $N S^{a)}$ \\
\hline
\end{tabular}

SJVP, vacuum phenomenon of the sacroiliac joint; PI, pelvic incidence; SD, standard deviation; NS, not significant; PT, pelvic tilt; SS, sacral slope; LL, lumbar lordosis.

a) Mann-Whitney U test.

of maximal load [11]. It is likely to incur more degenerative changes than other weight-bearing joints. However, movement is restricted to a single axis of rotation and translation is impeded by strong ventral and dorsal sacroiliac ligaments. The SIJ is therefore an amphiarthrosis.

VP is a collection of gas within the joint space. Intervertebral VP is regarded as a reliable indicator of intervertebral disc degeneration and low back pain $[12,13]$. In vertebral fracture, VP is a sign of pseudarthrosis requiring surgical intervention. In this study, the overall prevalence of SJVP was $38.7 \%$, similar to that reported in a larger series (34\% of 652 patients) [8]. Over $90 \%$ of female subjects had SJVP compared with only $8.5 \%$ of male subjects. The female predominance was also consistent with the aforementioned report [8]. However, the sex difference in SJVP prevalence was larger in our study. The effects of estrogen and pregnancy on ligaments can explain this female predominance [14]. Moreover, women show degenerative changes in the SIJ earlier than men [15].

While few male subjects had SJVP, those that did exhibit it had significantly lower PI than male subjects without it. As PI is an anatomical parameter (in other words, the angle of the sacrum at which it is docked to the pelvic bone), our results suggest that lower PI may cause biomechanical overloading on the SIJ specifically in men, and result in SJVP. The inclination angle of the SIJ articular surface correlated closely with PI in each individual. Individuals with lower PI have a more vertical sacrum, leading to greater stress or rotational force on the anterior part of the SIJ, which is a synovial joint with little gliding and rotatory movement. Numerous studies have been conducted on SIJ biomechanics, including finite element analysis of SIJ motions under different conditions [16-19]. However, there is no finite element model that verifies the effect of sacral inclination angle on SIJ stress distribution.

In this study, we reported for the first time the clinical relevance of SJVP using the RDQ, a widely used health status measure for low back pain, and we found no relationship between SJVP and low back pain.

The limitations of this study include the measurement of positional parameters (PT, SS, and LL) in CT images obtained in the supine position that may not reflect standing alignment. Although these lumbopelvic parameters did not differ markedly from those in other reports [20], there is likely to be a discrepancy in lumbopelvic positional parameters between the standing and supine positions [21]. Thus, a future analysis of the relationship between standing spinal alignment and presence of SJVP is desirable. Second, our study lacked clinical information about childbearing and age-related analysis from serial imaging, which could help explain the sex differences in SJVP.

\section{Conclusions}

Vacuum phenomenon of the SIJ was common in women but not in men, with only $8.5 \%$ affected. Men with SJVP showed significantly lower PI than men without SJVP. These data suggest that differences in sacropelvic morphology may influence the biomechanical environment, leading to SJVP in men. The presence of SJVP did not affect the JOA score or RDQ score.

\section{Conflict of Interest}

No potential conflict of interest relevant to this article was reported.

\section{References}

1. Glassman SD, Bridwell K, Dimar JR, Horton W, Berven S, Schwab F. The impact of positive sagittal balance in adult spinal deformity. Spine (Phila Pa 1976) 
2005;30:2024-9.

2. Lafage V, Schwab F, Patel A, Hawkinson N, Farcy JP. Pelvic tilt and truncal inclination: two key radiographic parameters in the setting of adults with spinal deformity. Spine (Phila Pa 1976) 2009;34:E599606.

3. Lazennec JY, Ramare S, Arafati N, et al. Sagittal alignment in lumbosacral fusion: relations between radiological parameters and pain. Eur Spine J 2000;9:4755.

4. Schwab F, Farcy JP, Bridwell K, et al. A clinical impact classification of scoliosis in the adult. Spine (Phila Pa 1976) 2006;31:2109-14.

5. Legaye J, Duval-Beaupere G, Hecquet J, Marty C. Pelvic incidence: a fundamental pelvic parameter for three-dimensional regulation of spinal sagittal curves. Eur Spine J 1998;7:99-103.

6. Gohil I, Vilensky JA, Weber EC. Vacuum phenomenon: clinical relevance. Clin Anat 2014;27:455-62.

7. Sturesson B, Uden A, Vleeming A. A radiostereometric analysis of movements of the sacroiliac joints during the standing hip flexion test. Spine (Phila $\mathrm{Pa}$ 1976) 2000;25:364-8.

8. Lo SS, Atceken Z, Carone M, Yousem DM. Sacroiliac joint vacuum phenomenon: underreported finding. Clin Imaging 2011;35:465-9.

9. Yetkin Z, Chintapalli K, Daniels DL, Haughton VM. Gas in spinal articulations. Neuroradiology 1986;28: 150-3.

10. Dietrichs E. Anatomy of the pelvic joints: a review. Scand J Rheumatol Suppl 1991;88:4-6.

11. Cusi MF. Paradigm for assessment and treatment of SIJ mechanical dysfunction. J Bodyw Mov Ther 2010; 14:152-61.

12. Morishita K, Kasai Y, Uchida A. Clinical symptoms of patients with intervertebral vacuum phenomenon.
Neurologist 2008;14:37-9.

13. Kasai Y, Takegami K, Uchida A. Change of barometric pressure influences low back pain in patients with vacuum phenomenon within lumbar intervertebral disc. J Spinal Disord Tech 2002;15:290-3.

14. Garagiola DM, Tarver RD, Gibson L, Rogers RE, Wass JL. Anatomic changes in the pelvis after uncomplicated vaginal delivery: a CT study on 14 women. AJR Am J Roentgenol 1989;153:1239-41.

15. Shibata Y, Shirai Y, Miyamoto M. The aging process in the sacroiliac joint: helical computed tomography analysis. J Orthop Sci 2002;7:12-8.

16. Kiapour A, Abdelgawad AA, Goel VK, Souccar A, Terai T, Ebraheim NA. Relationship between limb length discrepancy and load distribution across the sacroiliac joint: a finite element study. J Orthop Res 2012;30:1577-80.

17. Ivanov AA, Kiapour A, Ebraheim NA, Goel V. Lumbar fusion leads to increases in angular motion and stress across sacroiliac joint: a finite element study. Spine (Phila Pa 1976) 2009;34:E162-9.

18. Zheng N, Watson LG, Yong-Hing K. Biomechanical modelling of the human sacroiliac joint. Med Biol Eng Comput 1997;35:77-82.

19. García JM, Doblare M, Seral B, Seral F, Palanca D, Gracia L. Three-dimensional finite element analysis of several internal and external pelvis fixations. J Biomech Eng 2000;122:516-22.

20. Endo K, Suzuki H, Nishimura H, Tanaka H, Shishido T, Yamamoto K. Characteristics of sagittal spino-pelvic alignment in Japanese young adults. Asian Spine J 2014;8:599-604.

21. Lee ES, Ko CW, Suh SW, Kumar S, Kang IK, Yang JH. The effect of age on sagittal plane profile of the lumbar spine according to standing, supine, and various sitting positions. J Orthop Surg Res 2014;9:11. 